\title{
BMJ Open Using digital notifications to improve attendance in clinic: systematic review and meta-analysis
}

\author{
Dan Robotham, ${ }^{1}$ Safarina Satkunanathan, ${ }^{1}$ John Reynolds, ${ }^{2}$ Daniel Stahl, ${ }^{1}$ \\ Til Wykes ${ }^{1,2}$
}

To cite: Robotham D,

Satkunanathan S, Reynolds J, et al. Using digital notifications to improve attendance in clinic: systematic review and metaanalysis. BMJ Open 2016;6: e012116. doi:10.1136/ bmjopen-2016-012116

- Prepublication history and additional material is available. To view please visit the journal (http://dx.doi.org/ 10.1136/bmjopen-2016012116).

Received 31 March 2016 Revised 15 August 2016 Accepted 1 September 2016

CrossMark

\footnotetext{
${ }^{1}$ Institute of Psychiatry, Psychology \& Neuroscience, King's College London (KCL), London, UK

${ }^{2}$ South London and Maudsley NHS Foundation Trust, London, UK
}

Correspondence to Dr Dan Robotham; Dan.Robotham@kcl.ac.uk

\section{ABSTRACT}

Objectives: Assess the impact of text-based electronic notifications on improving clinic attendance, in relation to study quality (according to risk of bias), and to assess simple ways in which notifications can be optimised (ie, impact of multiple notifications).

Design: Systematic review, study quality appraisal assessing risk of bias, data synthesised in metaanalyses.

Data sources: MEDLINE, EMBASE, PsycINFO, Web of Science and Cochrane Database of Systematic Reviews (01.01.05 until 25.4.15). A systematic search to discover all studies containing quantitative data for synthesis into meta-analyses.

Eligibility criteria: Studies examining the effect of text-based electronic notifications on prescheduled appointment attendance in healthcare settings. Primary analysis included experimental studies where randomisation was used to define allocation to intervention and where a control group consisting of 'no reminders' was used. Secondary meta-analysis included studies comparing text reminders with voice reminders. Studies lacking sufficient information for inclusion (after attempting to contact study authors) were excluded.

Outcome measures: Primary outcomes were rate of attendance/non-attendance at healthcare appointments. Secondary outcome was rate of rescheduled and cancelled appointments.

Results: 26 articles were included. 21 included in the primary meta-analysis (8345 patients receiving electronic text notifications, 7731 patients receiving no notifications). Studies were included from Europe (9), Asia (7), Africa (2), Australia (2) and America (1). Patients who received notifications were $23 \%$ more likely to attend clinic than those who received no notification (risk ratio $=1.23,67 \%$ vs $54 \%$ ). Those receiving notifications were $25 \%$ less likely to 'no show' for appointments (risk ratio=.75, $15 \%$ vs $21 \%$ ). Results were similar when accounting for risk of bias, region and publication year. Multiple notifications were significantly more effective at improving attendance than single notifications. Voice notifications appeared more effective than text notifications at improving attendance.

Conclusions: Electronic text notifications improve attendance and reduce no shows across healthcare settings. Sending multiple notifications could improve attendance further.

\section{Strengths and limitations of this study}

- Updates and appraises the evidence for how electronic text notifications impact on appointment attendance.

- Assesses study quality using a risk of bias framework.

- Large number of participants means that the impact of high quality studies can be considered.

- Tests the effect of multiple notifications, one way in which notifications can be optimised.

\section{INTRODUCTION}

Reducing the number of missed healthcare appointments improves the efficiency of health services. Missing healthcare appointments without cancelling in advance results in a 'no show', a vacant appointment slot that cannot be offered to others. In 2015 , the UK Secretary of State for Health estimated that missed general practitioner (GP) and hospital appointments cost the National Health Service (NHS) an estimated $£ 912 \mathrm{~m}$ per year ${ }^{1}$ and most appointments are missed due to simple reasons such as forgetfulness. ${ }^{2} 3$ Missed appointments and no shows are more problematic in some areas of healthcare than others, for example, attendance is poor in community mental health settings which can have subsequent effects on care. ${ }^{4}$

'No shows' can be reduced by reminding patients about their appointment in advance. The simplest way to do this is through electronic text notifications to patients' phones. Currently, there are as many mobile subscriptions as people in the world. ${ }^{6}$ In $2014,93 \%$ of UK adults owned a mobile phone, with $61 \%$ having a smartphone (a 10\% increase from 2013; Ofcom, 2014). In the last two decades, the service of sending text messages from mobile phones has dramatically changed the way in which people communicate. The number of messages sent has increased threefold to >150 billion between 2006 and 2011. ${ }^{7}$ 
This form of communication is acceptable to the public and has been harnessed by healthcare providers to remind patients about their appointments.

For the purposes of this review, the term 'electronic text notifications' refers to written messages sent from a service provider to a patient, in order to help patients remember, cancel or reschedule healthcare appointments. Notifications can be sent to patients' phones by text message, email or instant messaging applications. They cost little and can be delivered almost instantly. ${ }^{8}$ Unlike voice notifications, patients are able to reread and refer back to text notifications at their own convenience, and they may be perceived as less intrusive. ${ }^{9}$ They are used throughout the world across healthcare settings, with studies and reviews demonstrating increased appointment attendance. ${ }^{10-13}$ A recent meta-analysis and systematic review showed $50 \%$ improvements in attendance (relative to when no notification was provided) $;{ }^{14}$ since this review was published, the use of technology is even more prevalent, with the use of smartphones almost doubling in the USA, (from $35 \%$ to $64 \%$ among adults). ${ }^{15}$

No-one has yet assessed the effectiveness of the intervention with regard to study quality/risk of bias. The large number of studies now available also allows an exploration of other potential predictive variables such as year of publication and geographical region. Similarly, there is little evidence on how to optimise electronic text notifications, specifically, whether the effect of multiple notifications is greater than the effect of a single notification and whether text notifications are as effective as voice notifications. This paper reviews and critically appraises the updated evidence for electronic text notifications and begins to answer such questions.

\section{Aim}

This review explores how much electronic text notifications improve attendance at healthcare appointments.

\section{METHODS}

\section{Types of studies}

We included all experimental studies containing quantitative data to be synthesised into a meta-analysis, in which randomisation was used to define allocation to the intervention. We included all studies published in the last 10 years (January 2005 until April 2015), including data from conference presentations where full published studies were unavailable. Where the same data had been published in two publications, the article with complete data was favoured (usually the later publication). Studies published prior to 2005 were excluded. The rationale is that mobile phone ownership was limited (and thus unrepresentative) prior to this date. No published protocol exists for this review.

\section{Types of participants}

We included all participants in studies which contained data measuring the effect of electronic text notifications on scheduled appointment attendance in any healthcare setting.

\section{Types of interventions}

We included studies examining the effect of electronic text notifications on the attendance of prescheduled healthcare appointments. Studies were only included in the primary analysis if they included a control group which received 'no notifications'. In cases where studies had multiple comparison groups (eg, electronic text notifications vs voice notifications vs no notifications), the data from alternative intervention groups were included in a secondary analysis.

We excluded:

- Data relating to patients attending non-scheduled drop-in clinics or where patients were reminded to book future appointments, or health outcomes other than clinic attendance (eg, adherence to medication).

- Studies not published in the peer-reviewed literature or presented at academic conferences or which lacked sufficient information to be included in the meta-analysis after contacting study authors (ie, studies failing to report the number of patients allocated to receive an electronic text notifications intervention).

\section{Outcomes}

Primary outcomes were the rate of attendance/nonattendance at healthcare appointments. The secondary outcome was the rate of rescheduling/cancellation of appointments (as opposed to 'no show' appointments, where the patient does not attend or cancel).

In addition to the effects on the primary and secondary outcomes, we investigated (i) whether potential predictive variables such as study quality, year of publication or geographical region affected the results, (ii) whether the number and timing of notifications affected the outcome, (iii) whether notifications had any effect in mental health settings which generally have the lowest attendance rates and (iv) how effective text reminders were in comparison to voice reminders (in studies which compared the two).

\section{Information sources}

The following bibliographic databases were searched (25.4.15): MEDLINE, EMBASE, PsycINFO, Web of Science and The Cochrane Database of Systematic Reviews (from January 2005 until the search date). A hand search was also conducted of the reference lists of included studies, which identified two additional studies. The key terms used in the electronic searches for each of the databases are shown in online supplementary appendices 1-5. Authors of studies were contacted for further information when it was not present in the published data, for example, to clarify the patient groups they had included in their study. 


\section{Data collection process}

Two reviewers (SS and JR) independently screened all the papers against the inclusion criteria. For the papers that met the inclusion criteria, the reviewers independently extracted information on the geographical location, clinic type, sample size, interventions and controls, study design, and comparison outcomes of attendance and non-attendance rates. Disagreements throughout this process were resolved by arbitration with a third reviewer (DR).

\section{Classification of data}

Articles were included and interpreted based on the outcome measures used. This fell into three categories; attendance in clinic, 'no show' rates in clinic and cancellations/rescheduled appointments in clinic. Attendance rate and 'no show' rate were examined separately. Although there are many similarities between these outcomes, they cannot be assumed as equivalent as some unattended appointments may be cancelled or rescheduled in advance, in which case they are not classifiable as 'no shows'. For those studies that measured attendance as a primary outcome measure, it was not possible to separate the proportion of 'no shows' and the proportion of cancellations.

\section{Assessment of risk of bias}

Studies were appraised using Cochrane Handbook for Systematic Reviews of Interventions. ${ }^{16}$ For each study which was to be included in the primary analysis, two reviewers (SS and JR) independently assessed the risk of bias. Each domain was judged as 'low', 'high' or 'unclear' risk (when insufficient information was provided to permit judgement). The agreement rate between the raters was $79 \% \quad(\kappa=0.58)$, a moderate level of agreement. Discrepancies between ratings were resolved by discussion with the third reviewer (DR).

All authors were contacted and asked for comments or clarifications on the risk of bias rating. The authors of 14 studies responded; changes were discussed with the reviewers. One or more changes were made to the ratings of seven studies. The most common reason for changing the rating was gaining access to study protocols. Studies were classified as either 'at risk' of bias or 'at low risk' of bias.

\section{Summary measures}

The principle summary measure was risk difference between those who attended appointments compared with those who missed their appointment (expressed as a percentage); risk ratios were also calculated for the primary outcome. We compared those groups in which patients had received an electronic text notification with groups who received no notification.

We combined the results of participants receiving electronic text notification 'intervention' from all included studies. These were compared against 'control' participants who received no notification. The percentages of the primary outcome measure (attendance, 'no shows' and appointment cancellation) for all known intervention groups and control groups were extracted. Some studies presented data from multiple intervention groups (eg, from different clinics). In these cases, the intervention groups were the unit of analysis rather than the study itself, for example. ${ }^{17}$ A secondary meta-analysis pooled the data from studies comparing electronic text notifications against voice reminder notifications.

\section{Synthesis of results}

Meta-analyses were conducted to determine the pooled effect size relating to intervention versus control groups using a random effects model. This is more realistic than fixed-effect meta-analyses in this situation due to the variety of populations and settings between studies. ${ }^{18}$ The primary meta-analysis was split by the three possible outcomes (i) attendance, (ii) 'no shows' and (iii) appointment cancellation. Pooled relative rates with exact Clopper-Pearson 95\% confidence limits are presented. The risk ratio and the risk difference were calculated for included studies along with their CIs (at 95\%), in order to calculate overall effect sizes for the intervention group and control group. Here, the risk ratio is the ratio of the probability of a positive event occurring in the intervention group to the probability of the event occurring in the control group. Interstudy heterogeneity was calculated using the $\mathrm{I}^{2}$ statistic $(\geq 50 \%$ indicated heterogeneity). Heterogeneity was investigated by conducting meta-regressions to examine the influence of risk of bias, year of publication and geographical region. We also investigated the impact of multiple notifications in comparison to single notifications.

We investigated whether any study had a large influence on the pooled estimate in sensitivity analyses by re-estimating meta-analysis omitting each study in turn using Stata's (V.11.2, StataCorp, College Station, Texas, the USA) 'metainf' command. ${ }^{19}$

Publication bias was assessed by visual inspections of funnel plots, Egger's test and using a non-parametric 'trim and fill' method. ${ }^{20}$ If the conclusion of the meta-analysis remains unchanged following adjustment for the publication bias using the trim and fill method, the results can be considered as robust, excluding publication bias. All analyses were carried out in Stata (V.11.2, StataCorp).

\section{RESULTS}

\section{Study selection}

After duplicates had been removed, 3981 articles were screened. Of these, 3910 articles were excluded based on the abstract alone; these studies clearly did not meet the inclusion criteria. A further 45 articles were excluded after reading full-text articles. The primary reason for exclusion in each case were as follows: nonrandomised study $(n=23)$, examined the effects of electronic text notifications on reminding patients to take 
medication or to make appointments (rather than to attend) $(n=3)$, only collecting data on preference for notifications rather than information about their effect on attendance $(n=9)$, only providing secondary data or where the same data had been assimilated in another included paper $(n=6)$, exclusively using other notification systems such automated call backs $(n=2)$ or lacking sufficient information to be included in the meta-analysis after we attempted to contact authors $(n=2)$. In total, 26 articles met the study inclusion criteria and were included in the systematic review. Of these, five studies were excluded from the primary meta-analysis because they lacked a 'no intervention' control group. Instead, they were included in the secondary meta-analysis comparing electronic notifications against voice notifications. The Preferred Reporting Items for Systematic review and Meta-Analysis (PRISMA) flow chart (figure 1) ${ }^{21}$ describes the process in which studies were included and excluded:

\section{Study characteristics for primary meta-analysis}

Of all data included in the primary meta-analysis, 12 studies used attendances as an outcome measure, 16 measured 'no show' rates and 3 measured cancellation. A total of 8345 patients received electronic text notifications, and 7731 patients received no notification. Randomised studies typically compared attendance or 'no show' rates of those who received electronic text notifications with those who did not receive notifications.

Studies spanned Europe (nine), Asia (seven), America (one), Africa (two) and Australia (two). The most common study context was primary care/general physical healthcare (11); followed by sexual health (3), dental care (2), mental health (2), and paediatrics (2). Other types of health intervention included paediatrics, postnatal care, blood donation, optometry, chronic illness, allergies and mixed contexts.

All included studies used short message service (SMS) notifications; one study used this in conjunction with follow-up phone calls and postal notifications. A typical example of an electronic text notification informed the patient of the time and date of the appointment and asked them to respond if they could not come to the appointment, for example: 'You have an appointment on... (date) at ... (time) with $\mathrm{Dr} \ldots$ (name) Please answer NO if you do not intend to come'.

Studies differed in the number and in the timing of notifications sent to patients. The majority of studies $(n=13)$ sent only one notification. Two notifications were sent in two studies; more than two notifications were sent in three studies, one study used voice notifications followed up with electronic text notifications (this was classified as a study in which 'more than one notification' was sent). One study did not provide this information. In one anomalous study, notifications were sent on every day for 30 days prior to an appointment.
Figure 1 PRISMA flow chart. PRISMA, Preferred Reporting Items for Systematic review and Meta-Analysis.
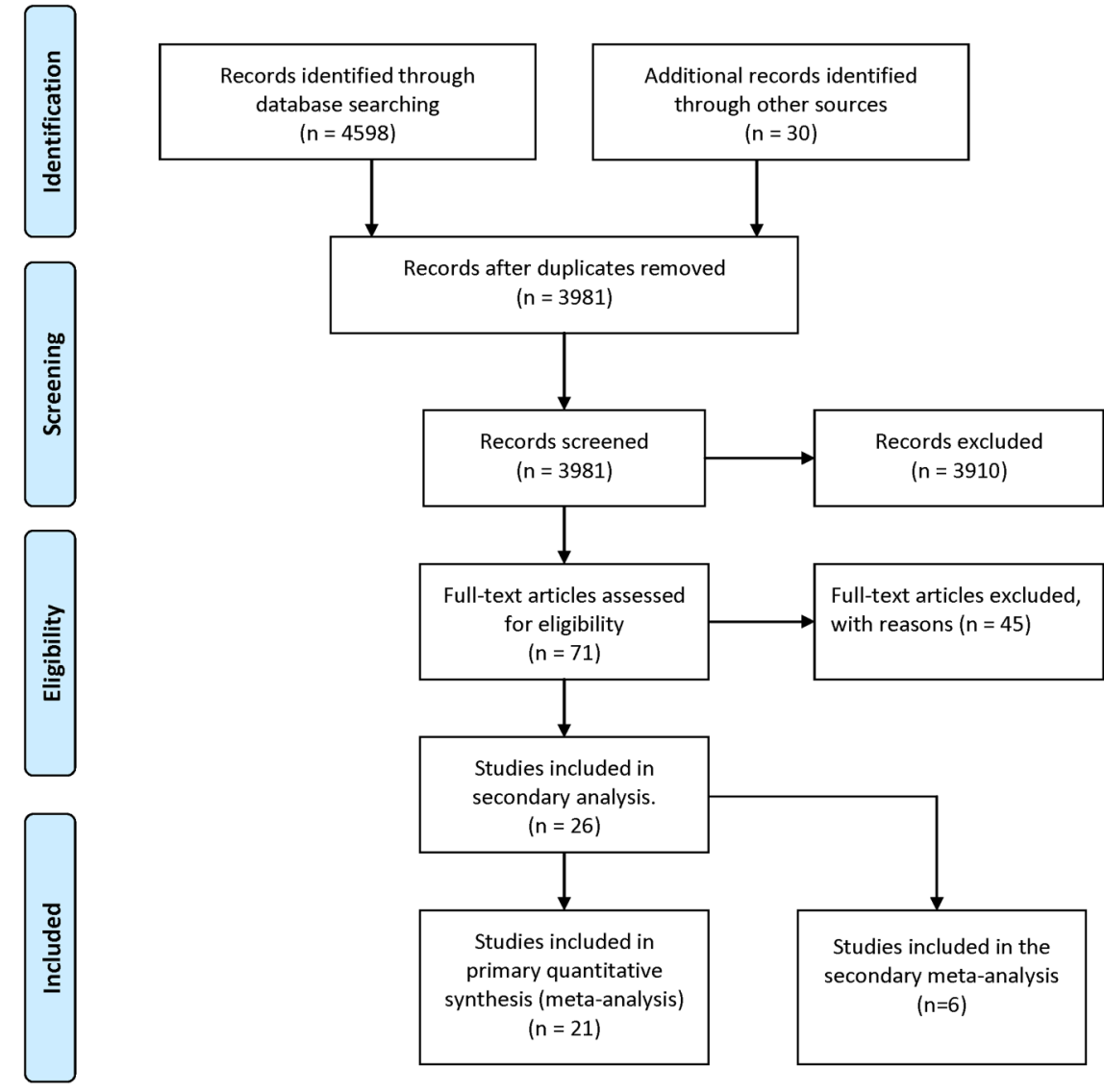
Table 1 Studies included in meta-analyses

\begin{tabular}{|c|c|c|c|c|c|c|}
\hline Study & $\begin{array}{l}\text { Subject area; } \\
\text { study design; } \\
\text { country }\end{array}$ & Participants & $\begin{array}{l}\text { Intervention and } \\
\text { comparator }\end{array}$ & Notification characteristics & $\begin{array}{l}\text { Outcome } \\
\text { measures }\end{array}$ & Prevalence rates \\
\hline $\begin{array}{l}\text { Arora et } a R^{2} \\
(2015)\end{array}$ & $\begin{array}{l}\text { Primary care; } \\
\text { randomised; the } \\
\text { USA }\end{array}$ & $\begin{array}{l}\text { In total, } 328 \text { patients from the } \\
\text { Emergency Department at Los } \\
\text { Angeles County USC Medical } \\
\text { Center. }\end{array}$ & $\begin{array}{l}\text { Interventions: SMS } \\
\text { reminders } \\
\text { Control: no } \\
\text { reminders }\end{array}$ & $\begin{array}{l}\text { Three reminders: } 7,3 \text { and } \\
1 \text { days before appointment. }\end{array}$ & Attendance rate & $\begin{array}{l}\text { Int }=73 \% \\
\text { Control=62\% }\end{array}$ \\
\hline $\begin{array}{l}\text { Bigna et } a f^{3} \\
\text { (2014) }\end{array}$ & $\begin{array}{l}\text { Preventative } \\
\text { medicine; } \\
\text { randomised; } \\
\text { Cameroon }\end{array}$ & $\begin{array}{l}\text { In total, } 242 \text { adult-child (carer } \\
\text { patient) pairs who were infected } \\
\text { with or had been exposed to HIV } \\
\text { attending clinics in urban, } \\
\text { semiurban and rural settings. }\end{array}$ & $\begin{array}{l}\text { Intervention: SMS } \\
\text { reminders } \\
\text { Control: no } \\
\text { reminders }\end{array}$ & $\begin{array}{l}\text { One reminder: } 2 \text { or } 3 \text { days } \\
\text { before appointment }\end{array}$ & Attendance rate & $\begin{array}{l}\text { Attendance } \\
\text { Int }=75 \% \\
\text { Control }=51 \%\end{array}$ \\
\hline $\begin{array}{l}\text { Bos et a } P^{4} \\
(2005)\end{array}$ & $\begin{array}{l}\text { Dental care; } \\
\text { randomised; the } \\
\text { Netherlands }\end{array}$ & $\begin{array}{l}\text { In total, } 143 \text { patients attending } \\
\text { the orthodontic department of the } \\
\text { Academic Centre of Dentistry in } \\
\text { Amsterdam. }\end{array}$ & $\begin{array}{l}\text { Intervention: SMS } \\
\text { reminders } \\
\text { Control: no } \\
\text { reminders }\end{array}$ & $\begin{array}{l}\text { One reminder: } 1 \text { day before } \\
\text { appointment }\end{array}$ & $\begin{array}{l}\text { Attendance rate, } \\
\text { non-attendance rate, } \\
\text { cancellation/ } \\
\text { reschedule rate }\end{array}$ & $\begin{array}{l}\text { Attendance } \\
\text { Int }=3 \% \\
\text { Control=7\% } \\
\text { 'No show' } \\
\text { Int }=82 \% \\
\text { Control=84\% } \\
\text { Cancellations } \\
\text { Int }=16 \% \\
\text { Control=10\% }\end{array}$ \\
\hline $\begin{array}{l}\text { Chen et } a f^{25} \\
(2008)\end{array}$ & $\begin{array}{l}\text { Health promotion; } \\
\text { randomised; } \\
\text { China }\end{array}$ & $\begin{array}{l}\text { In total, } 1891 \text { adults who had } \\
\text { scheduled appointments within } \\
72 \text { hours to } 2 \text { months from } \\
\text { recruitment. }\end{array}$ & $\begin{array}{l}\text { Intervention: SMS } \\
\text { reminders } \\
\text { Control: no } \\
\text { reminders }\end{array}$ & $\begin{array}{l}\text { One reminder: } 3 \text { days before } \\
\text { appointment }\end{array}$ & Attendance rate & $\begin{array}{l}\text { Int }=88 \% \\
\text { Control }=81 \%\end{array}$ \\
\hline $\begin{array}{l}\text { Cho et } a \text { P }^{6} \\
(2010)\end{array}$ & $\begin{array}{l}\text { Health promotion; } \\
\text { randomised; } \\
\text { South Korea }\end{array}$ & $\begin{array}{l}\text { In total, } 918 \text { adults attending } \\
\text { family practices for lipid lowering. }\end{array}$ & $\begin{array}{l}\text { Intervention: SMS } \\
\text { reminders } \\
\text { Control: no } \\
\text { reminders } \\
\text { Note: a third group of } \\
\text { participants received } \\
\text { postal reminder (not } \\
\text { included) }\end{array}$ & $\begin{array}{l}\text { One reminder: } 8 \text { weeks } \\
\text { before appointment (week } \\
16 \text { of } 24 \text { ). }\end{array}$ & Attendance rate & $\begin{array}{l}\text { Int }=76 \% \\
\text { Control }=72 \%\end{array}$ \\
\hline $\begin{array}{l}\text { Clough and } \\
\text { Casey }^{27} \\
(2014)\end{array}$ & $\begin{array}{l}\text { Mental health; } \\
\text { Randomised; } \\
\text { Australia }\end{array}$ & $\begin{array}{l}140 \text { consecutive adults seeking } \\
\text { psychotherapeutic treatment at } \\
\text { an outpatient psychology clinic at } \\
\text { Brisbane University. }\end{array}$ & $\begin{array}{l}\text { Intervention: SMS } \\
\text { reminders } \\
\text { Control: No } \\
\text { reminders }\end{array}$ & $\begin{array}{l}\text { One reminder: } 1 \text { day before } \\
\text { appointment (between 8:30 } \\
\text { and 9:00) }\end{array}$ & $\begin{array}{l}\text { Attendance rate, } \\
\text { Non-attendance } \\
\text { rate, Cancellation/ } \\
\text { reschedule rate }\end{array}$ & $\begin{array}{l}\text { Attendance } \\
\text { Int=89\% } \\
\text { Control=91\% 'No } \\
\text { show' } \\
\text { Int }=7 \% \\
\text { Control=6\% } \\
\text { Cancellations } \\
\text { Int=4\% } \\
\text { Control=3\% }\end{array}$ \\
\hline
\end{tabular}

In total, 328 patients from the comparator

Three reminder

measures

Int $73 \%$

1 days before appointment.

Int $=73 \%$

One reminder: 2 or 3 days

patient) pairs who were infected

with or had been exposed to HIV

Control: $n$

semiurban and rural settings.

In total, 143 patients attending

reminders

Control: no

non-attendance rate

Int $=3 \%$

cancellation/

Control=7\%

'nt=82\%

Control $=84 \%$

Int $=16 \%$

Control $=10 \%$

Int $=88 \%$

reminders

appointment 
Table 1 Continued

\begin{tabular}{|c|c|c|c|c|c|c|}
\hline Study & $\begin{array}{l}\text { Subject area; } \\
\text { study design; } \\
\text { country }\end{array}$ & Participants & $\begin{array}{l}\text { Intervention and } \\
\text { comparator }\end{array}$ & Notification characteristics & $\begin{array}{l}\text { Outcome } \\
\text { measures }\end{array}$ & Prevalence rates \\
\hline $\begin{array}{l}\text { Narring et } a \beta^{34} \\
(2013)\end{array}$ & $\begin{array}{l}\text { Youth clinic; } \\
\text { randomised; } \\
\text { Switzerland }\end{array}$ & $\begin{array}{l}\text { In total, } 616 \text { patients aged } 12- \\
24 \text { years with primary care } \\
\text { appointments at a } \\
\text { multidisciplinary clinic. Plus } 203 \\
\text { patients with gynaecological } \\
\text { appointments and } 165 \text { patients } \\
\text { with mental healthcare } \\
\text { appointments at a } \\
\text { multidisciplinary clinic. }\end{array}$ & $\begin{array}{l}\text { Intervention: SMS } \\
\text { reminders } \\
\text { Control: no } \\
\text { reminders }\end{array}$ & $\begin{array}{l}\text { One reminder: } 8: 00-11: 00 \\
\text { the day before appointment }\end{array}$ & Non-attendance rate & $\begin{array}{l}\text { Int }=20 \% \\
\text { Control=20\% }\end{array}$ \\
\hline $\begin{array}{l}\text { Odeny et } a{ }^{\beta 5} \\
(2012)\end{array}$ & $\begin{array}{l}\text { Sexual health; } \\
\text { randomised; } \\
\text { Kenya }\end{array}$ & $\begin{array}{l}\text { In total, } 1188 \text { men undergoing } \\
\text { circumcision at any of } 12 \text { sites in } \\
\text { Nyanza province. }\end{array}$ & $\begin{array}{l}\text { Intervention: SMS } \\
\text { reminders } \\
\text { Control: no } \\
\text { reminders }\end{array}$ & $\begin{array}{l}\text { Seven reminders: daily for } \\
7 \text { days before appointment }\end{array}$ & Non-attendance rate & $\begin{array}{l}\text { Int }=35 \% \\
\text { Control=40\% }\end{array}$ \\
\hline $\begin{array}{l}\text { Perron et }\left.a\right|^{36} \\
(2010)\end{array}$ & $\begin{array}{l}\text { Sexual health; } \\
\text { randomised; } \\
\text { Switzerland }\end{array}$ & $\begin{array}{l}\text { In total, } 2123 \text { patients scheduled } \\
\text { to attend primary care clinic and } \\
\text { ambulatory HIV clinic of the } \\
\text { Geneva University Hospitals, } \\
\text { between April and June } 2008\end{array}$ & $\begin{array}{l}\text { Intervention: a } \\
\text { combination of } \\
\text { phone, SMS and } \\
\text { postal reminders } \\
\text { Control: no } \\
\text { reminders }\end{array}$ & $\begin{array}{l}\text { Sequential intervention, one } \\
\text { phone call } 48 \text { hours before } \\
\text { appointment. If phone was } \\
\text { not answered after three } \\
\text { attempts, either text } \\
\text { message sent or postal } \\
\text { message if they did not have } \\
\text { a phone. }\end{array}$ & Non-attendance rate & $\begin{array}{l}\text { Int }=8 \% \\
\text { Control=11\% }\end{array}$ \\
\hline $\begin{array}{l}\text { Prasad and } \\
\text { Anand }^{37} \\
(2012)\end{array}$ & $\begin{array}{l}\text { Dental care; } \\
\text { randomised; the } \\
\text { Netherlands }\end{array}$ & $\begin{array}{l}\text { In total, } 206 \text { patients who were } \\
\text { scheduled to attend four selected } \\
\text { departments from September } \\
2010 \text { to December } 2010\end{array}$ & $\begin{array}{l}\text { Intervention: SMS } \\
\text { reminders } \\
\text { Control: no } \\
\text { reminders }\end{array}$ & $\begin{array}{l}\text { Two reminders: } 24 \text { hours } \\
\text { before, and on day of } \\
\text { appointment }\end{array}$ & Attendance rate & $\begin{array}{l}\text { Int }=79 \% \\
\text { Control=36\% }\end{array}$ \\
\hline $\begin{array}{l}\text { Reeve-Mates } \\
\text { et al (under } \\
\text { review) }\end{array}$ & $\begin{array}{l}\text { Mental health, } \\
\text { randomised, UK }\end{array}$ & $\begin{array}{l}\text { In total, } 75 \text { patients attending } \\
\text { mental health services from } \\
\text { January to July } 2014 \text {. }\end{array}$ & $\begin{array}{l}\text { Intervention: SMS } \\
\text { reminders } \\
\text { Control: no } \\
\text { reminders }\end{array}$ & $\begin{array}{l}\text { Two reminders: } 7 \text { days and } \\
1 \text { day before appointment }\end{array}$ & $\begin{array}{l}\text { Attendance rate, } \\
\text { non-attendance rate, } \\
\text { cancellation/ } \\
\text { reschedule rate }\end{array}$ & $\begin{array}{l}\text { Attendance } \\
\text { Int=70\% } \\
\text { Control=59\% } \\
\text { 'No show' } \\
\text { Int=6\% } \\
\text { Control=20\% } \\
\text { Cancellations } \\
\text { Int }=15 \% \\
\text { Control=13\% }\end{array}$ \\
\hline $\begin{array}{l}\text { Rutland et } a \beta^{\beta 8} \\
\text { (2012) }\end{array}$ & $\begin{array}{l}\text { Sexual health; } \\
\text { randomised; UK }\end{array}$ & $\begin{array}{l}\text { In total, } 252 \text { patients aged } \\
16-30 \text { years who booked an } \\
\text { appointment during the } 6 \text { month } \\
\text { study period. Only gave } \\
\text { intervention to people who had } \\
\text { missed appointments in the past. }\end{array}$ & $\begin{array}{l}\text { Intervention 1: SMS } \\
\text { reminders } \\
\text { Intervention 2: SMS } \\
\text { reminders plus } \\
\text { health promotion } \\
\text { Control: no } \\
\text { reminders }\end{array}$ & $\begin{array}{l}\text { One reminder: } 1 \text { week after } \\
\text { they had missed their initial } \\
\text { appointment (for attendance } \\
\text { within } 4 \text { weeks). }\end{array}$ & Non-attendance rate & $\begin{array}{l}\text { (Pooled) } \\
\text { Int }=12 \% \\
\text { Control=5\% }\end{array}$ \\
\hline
\end{tabular}


Table 1 Continued

\begin{tabular}{|c|c|c|c|c|c|c|}
\hline Study & $\begin{array}{l}\text { Subject area; } \\
\text { study design; } \\
\text { country }\end{array}$ & Participants & $\begin{array}{l}\text { Intervention and } \\
\text { comparator }\end{array}$ & Notification characteristics & $\begin{array}{l}\text { Outcome } \\
\text { measures }\end{array}$ & Prevalence rates \\
\hline $\begin{array}{l}\text { Taylor et } a \AA^{39} \\
\text { (2012) }\end{array}$ & $\begin{array}{l}\text { Physical therapy; } \\
\text { randomised; } \\
\text { Australia }\end{array}$ & $\begin{array}{l}\text { In total, } 696 \text { participants who had } \\
\text { an appointment in a physical } \\
\text { therapy outpatient clinic at one of } \\
\text { the two participating clinics. }\end{array}$ & $\begin{array}{l}\text { Intervention: SMS } \\
\text { reminders } \\
\text { Control: no } \\
\text { reminders }\end{array}$ & $\begin{array}{l}\text { One reminder: } 2 \text { days before } \\
\text { appointment (if made } 3+ \\
\text { days prior), } 1 \text { day before } \\
\text { appointment (if made } 2 \text { days } \\
\text { prior). }\end{array}$ & Non-attendance rate & $\begin{array}{l}\text { Int }=11 \% \\
\text { Control=16\% }\end{array}$ \\
\hline $\begin{array}{l}\text { Wang et } a f^{40} \\
(2014)\end{array}$ & $\begin{array}{l}\text { Allergic rhinitis; } \\
\text { randomised; } \\
\text { China }\end{array}$ & $\begin{array}{l}\text { In total, } 50 \text { patients with a history } \\
\text { of physician-diagnosed allergic } \\
\text { rhinitis who had an appointment } \\
\text { scheduled from December } 2011 \\
\text { to March } 2012 \text {. }\end{array}$ & $\begin{array}{l}\text { Intervention: SMS } \\
\text { reminders } \\
\text { Control: no } \\
\text { reminders }\end{array}$ & $\begin{array}{l}\text { A total of } 30 \text { reminders for } \\
\text { medication: daily reminder } \\
\text { for } 30 \text { days, at } 7: 00 \text { on } \\
\text { Monday to Friday and at } \\
\text { 9:00. on Saturday and } \\
\text { Sunday. }\end{array}$ & Attendance rate & $\begin{array}{l}\text { Int }=72 \% \\
\text { Control=40\% }\end{array}$ \\
\hline $\begin{array}{l}\text { Youssef, et al }{ }^{17} \\
(2014)\end{array}$ & $\begin{array}{l}\text { General health; } \\
\text { randomised; Saudi } \\
\text { Arabia }\end{array}$ & $\begin{array}{l}\text { In total, } 2297 \text { outpatients } \\
\text { attending one of four clinics at the } \\
\text { King Fahad teaching hospital } \\
\text { from April to June } 2011 \text {. }\end{array}$ & $\begin{array}{l}\text { Intervention: SMS } \\
\text { reminders } \\
\text { Control: no } \\
\text { reminders }\end{array}$ & $\begin{array}{l}\text { One reminder: } 48 \text { hours } \\
\text { before appointment. }\end{array}$ & Non-attendance rate & $\begin{array}{l}\text { (Pooled) } \\
\text { Int=27\% } \\
\text { Control=37\% }\end{array}$ \\
\hline \multicolumn{7}{|c|}{ Studies included in secondary meta-analysis } \\
\hline $\begin{array}{l}\text { Fung et } a f^{41} \\
(2009)\end{array}$ & $\begin{array}{l}\text { Blood donor; } \\
\text { randomised; the } \\
\text { USA }\end{array}$ & $\begin{array}{l}\text { In total, } 31 \text { repeat blood donors } \\
\text { who made donation appointments } \\
\text { in October } 2008 .\end{array}$ & $\begin{array}{l}\text { Intervention 1: SMS } \\
\text { reminders } \\
\text { Intervention 2: } \\
\text { telephone reminders }\end{array}$ & No information available & Attendance rate & Int $=56 \%$ \\
\hline $\begin{array}{l}\text { Liew et al (2009) } \\
\text { (also included in } \\
\text { primary anlaysis) }\end{array}$ & $\begin{array}{l}\text { Chronic illnesses; } \\
\text { randomised; } \\
\text { Malaysia }\end{array}$ & $\begin{array}{l}\text { In total, } 617 \text { patients requiring } \\
\text { chronic disease care from two } \\
\text { primary care clinics in Kuala } \\
\text { Lumpur. }\end{array}$ & $\begin{array}{l}\text { Intervention 1: SMS } \\
\text { reminders } \\
\text { Intervention 2: } \\
\text { telephone reminders } \\
\text { Control: no } \\
\text { reminders (included } \\
\text { in primary } \\
\text { meta-analysis) }\end{array}$ & $\begin{array}{l}\text { One reminder: } 24-48 \text { hours } \\
\text { before appointment }\end{array}$ & Non-attendance rate & $\begin{array}{l}\text { Int }=16 \% \\
\text { Control=23\% }\end{array}$ \\
\hline $\begin{array}{l}\text { Nelson et } a \digamma^{42} \\
(2011)\end{array}$ & $\begin{array}{l}\text { Paediatric } \\
\text { dentistry; } \\
\text { randomised; the } \\
\text { USA }\end{array}$ & $\begin{array}{l}\text { In total, } 318 \text { caregiver/child dyads } \\
\text { attending a paediatric dentistry } \\
\text { clinic at the University of } \\
\text { Washington, Seattle. }\end{array}$ & $\begin{array}{l}\text { Intervention 1: SMS } \\
\text { reminders } \\
\text { Intervention 2: } \\
\text { telephone reminders }\end{array}$ & $\begin{array}{l}\text { One reminder: } 48 \text { hours } \\
\text { before appointment }\end{array}$ & $\begin{array}{l}\text { Attendance rate } \\
\text { Non-attendance rate }\end{array}$ & $\begin{array}{l}\text { Attendance } \\
\text { Int }=82.3 \% \\
\text { 'No show' } \\
\text { Int }=17.7 \%\end{array}$ \\
\hline $\begin{array}{l}\text { Norton et } a f^{43} \\
(2014)\end{array}$ & $\begin{array}{l}\text { Sexual health; } \\
\text { randomised; the } \\
\text { USA }\end{array}$ & $\begin{array}{l}\text { In total, } 52 \text { adults from the Duke } \\
\text { University Medical Center } \\
\text { (Durham, NC) Adult Infectious } \\
\text { Diseases Clinic from June to } \\
\text { August } 2010 .\end{array}$ & $\begin{array}{l}\text { Intervention 1: SMS } \\
\text { reminders } \\
\text { Intervention 2: } \\
\text { telephone reminders }\end{array}$ & $\begin{array}{l}\text { One reminder: } 1 \text { day before } \\
\text { appointment }\end{array}$ & Attendance rate & Int $=72 \%$ \\
\hline
\end{tabular}




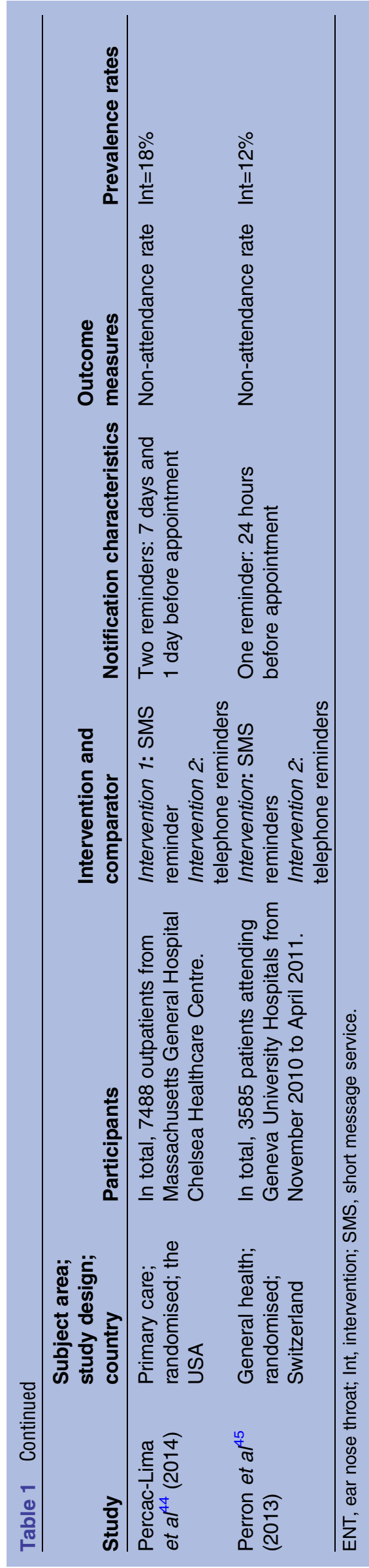

Regarding timing of messages, in nine studies notifications were sent 48 hours (or less) before the appointment. In three studies, notifications were sent over 48 hours before the appointment. In one study, participants were sent a notification 8 weeks prior to their appointment. Of the eight studies where two or more notifications were sent, the majority $(n=5)$ reminded patients before and after the 48 hour mark. Full details of individual studies are presented in table 1 .

\section{Study characteristics for secondary meta-analysis}

The secondary meta-analysis included six studies (only one of which had been included in the primary meta-analysis). Attendance rates were measured in three studies, 'no show' rates were measured in four. A total of 9885 patients received electronic text notifications, and 5076 patients received voice notifications. The studies were conducted in America (four), Europe (one) and Asia (one). Context included were as follows: primary care/general healthcare (two), sexual health (one), dental paediatrics (one), blood donation (one) and chronic illness (one).

\section{Risk of bias within and across studies}

The risk of bias within individual studies is presented in online supplementary appendix 6 . We used items of Cochrane's framework in judging the quality of the studies. The corresponding author of each article was sent their assessment to check and suggest revisions if necessary. Biases relating to blinding were considered of lesser importance in context of the intervention; participants cannot be blinded to a notification intervention, and outcome assessment is objective (ie, the participant either attended appointment or not). Random sequence generation and incomplete outcome data were considered important potential biases. The most common reason for 'unclear' bias was the unavailability of protocols.

\section{Primary meta-analysis results: main outcomes}

The pooled attendance rate was $67 \%(\mathrm{~N}=13$, CI $53 \%$ to $82 \%)$ for intervention groups and $54 \%(\mathrm{~N}=13$, CI $37 \%$ to $70 \%$ ) for control groups. The risk ratio was 1.23 (CI 1.10 to $1.38 ; \mathrm{N}=13, \mathrm{p}<0.01, \mathrm{I}^{2}=83 \%$ ), the risk difference was $13 \%$ in favour of the intervention group $(95 \%$ CI $6 \%$ to $\left.19 \% ; \mathrm{N}=13, \mathrm{p}<0.01, \mathrm{I}^{2}=82 \%\right)$. The pooled 'no show' rate was $15 \%(\mathrm{~N}=16$, CI $10 \%$ to $19 \%)$ for intervention groups and $21 \%(\mathrm{~N}=16$, CI $16 \%$ to $26 \%)$ for control groups. The risk ratio was 0.75 (CI 0.68 to 0.82 ; $\mathrm{N}=16, \mathrm{p}<0.01, \mathrm{I}^{2}=21 \%$ ), the risk difference was $5 \%$ in favour of the intervention group (95\% CI $-7 \%$ to $-3 \%$; $\mathrm{N}=16, \mathrm{p}<0.01, \quad \mathrm{I}^{2}=31 \%$ ). The percentage difference between intervention and control groups for each study is shown in figures 2 and 3.

The pooled cancellation rates were $11 \%$ for intervention $(\mathrm{N}=3$, CI $-2 \%$ to $19 \%)$ and $8 \%$ control $(\mathrm{N}=3$, CI $-1 \%$ to $14 \%)$ groups. The risk ratio was non-significant at $1.37\left(\mathrm{~N}=3, \mathrm{p}=0.34, \mathrm{I}^{2}<1\right)$ as was the $2 \%$ risk difference at $\left(\mathrm{N}=3, \mathrm{p}=0.4, \mathrm{I}^{2}<1\right)$. 


\section{attendance}

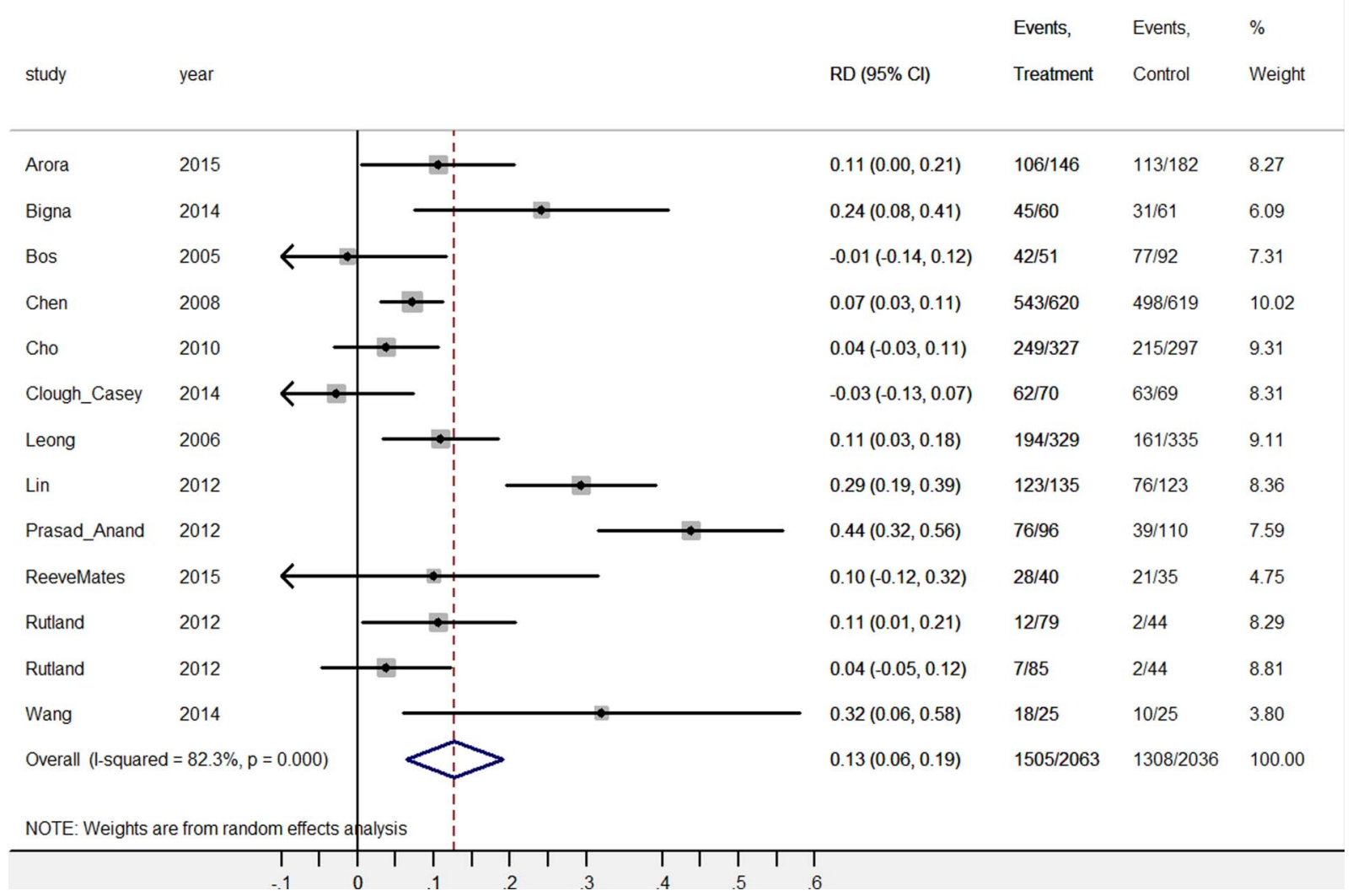

Figure 2 Effect of notifications on attendance rates. RD, risk difference.

Visual inspection of funnel plots (see figures 4 and 5 , see online supplementary appendix 7) revealed evidence of potential publication bias in attendance but little evidence of publication bias in 'no shows'. The trim and fill method revealed no missing studies. Egger's test was not significant in the meta-analyses.

\section{Assessing and identifying study heterogeneity}

Meta-regression compared the impact of the following potential predictive variables: risk of bias (high bias, low bias), number of notifications (one, multiple), year of publication (2005-2010, 2011-2015) and geographic region (Europe, Asia, other), where the 'other' category was created because there were few studies in the other continents.

Of these variables, the only significant finding was the effect of multiple reminders on appointment attendance (shown in table 2). Multiple notifications increased the risk of patients attending appointments by $25 \%$ (compared with $6 \%$ for patients receiving one notification), but multiple reminders did not make a significant difference in reducing 'no shows'. No significant effects were found for risk of bias $(p=0.88$ for attendance, $p=0.68$ for 'no shows'), age of study ( $\mathrm{p}=0.16$ for attendance, $\mathrm{p}=0.38$ for 'no shows') or geographic region $(\mathrm{F}=0.11, \mathrm{p}=0.9$ for attendance; $\mathrm{F}=1.6, \mathrm{p}=0.23$ for 'no shows'). No significant associations were found when all variables were pooled
( $\mathrm{F}=2.35 \mathrm{p}=0.15$ for attendance; $\mathrm{F}=0.66, \mathrm{p}=0.66$ for 'no shows').

\section{Sensitivity analyses}

In a sensitivity analysis in which the meta-analyses were repeated, excluding one study at a time to investigate the influence of each individual study on the overall meta-analysis summary did not reveal that any single study significantly affected the results.

\section{What happens in mental health?}

We pooled the results of three studies (182 participants received notifications, 197 did not). Two studies measured attendance and three measured 'no shows'. One took place in psychosis services, one in a university therapeutic context and one at a youth clinic. The pooled attendance rates were $85 \%$ for intervention $(\mathrm{N}=2$, CI $78 \%$ to $91 \%)$ and $87 \%$ for control $(\mathrm{N}=2$, CI $81 \%$ to $93 \%$ ). The risk ratio was 1.01 (CI 0.85 to 1.2 ; $\left.\mathrm{N}=2, \mathrm{p}=.92, \mathrm{I}^{2}=30 \%\right)$, the risk difference was $<1 \%(95 \%$ CI $-11 \%$ to $12 \%$; $\mathrm{N}=2, \mathrm{p}=0.93, \mathrm{I}^{2}=25 \%$ ). The pooled rate of 'no shows' was $7 \%$ for intervention $(\mathrm{N}=3$, CI $3 \%$ to $11 \%)$ and $13 \%$ for control ( $\mathrm{N}=3$, CI $4 \%$ to $22 \%)$. The risk ratio was 0.61 (CI 0.29 to $1.29 ; \mathrm{N}=3, \mathrm{p}=0.2, \mathrm{I}^{2}=21 \%$ ), the risk difference was $5 \%(95 \% \mathrm{CI}-14 \%$ to $4 \%$; $=3$, $\left.\mathrm{p}=0.26, \mathrm{I}^{2}=52 \%\right)$. 


\section{dna}

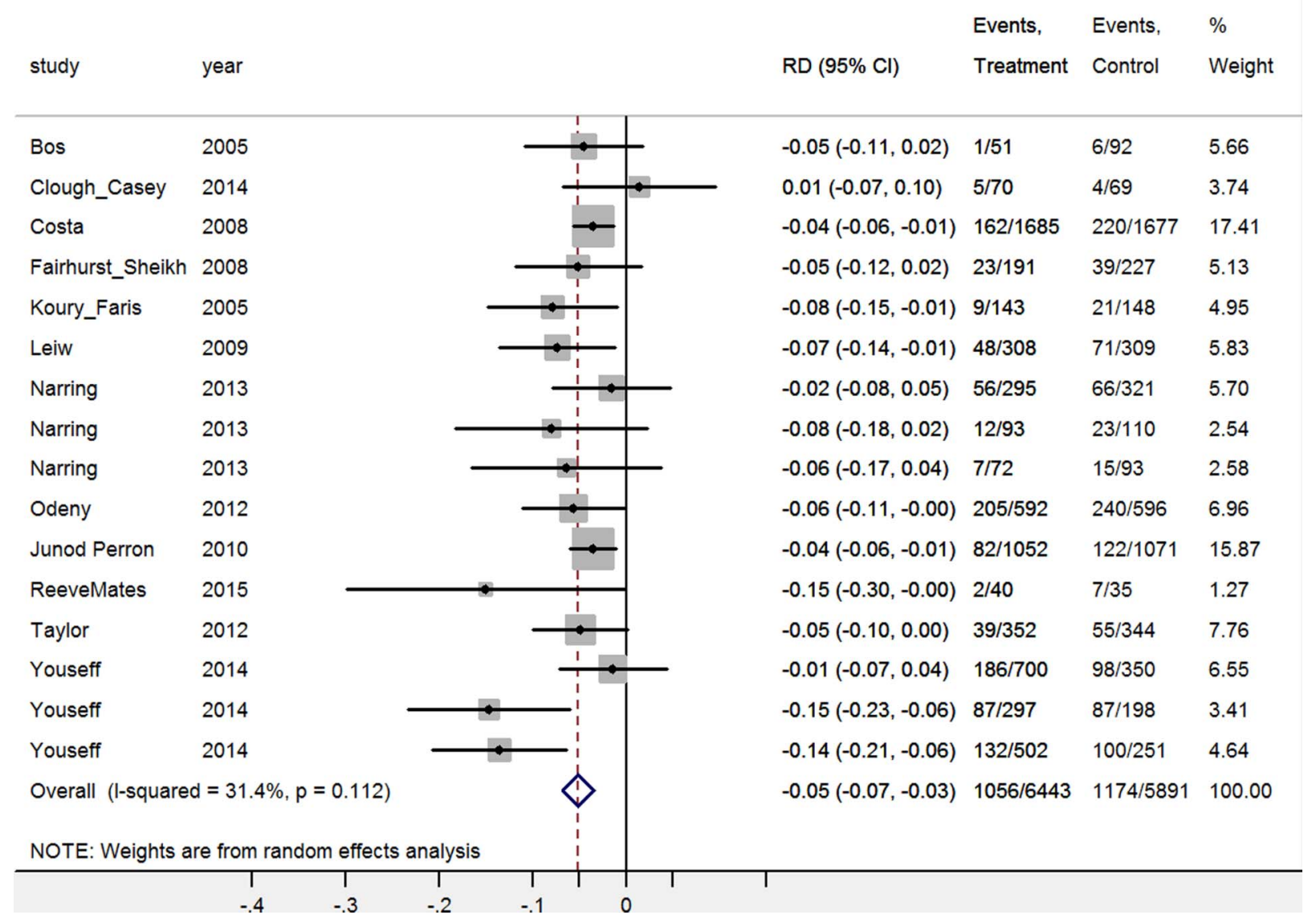

Figure 3 Effect of notifications on 'no show' rates. RD, risk difference.

How do electronic text notifications compare to voice notifications? (secondary meta-analysis)

The pooled attendance rate for the electronic text notifications was $74 \%(\mathrm{~N}=3$, CI $60 \%$ to $88 \%$ ) and $74 \%$ for voice notifications ( $\mathrm{N}=3$, CI $51 \%$ to $97 \%$ ). This difference was significant; however, the risk ratio was 0.90 (CI 0.82 to $0.98 ; \mathrm{N}=3, \mathrm{p}=0.01, \mathrm{I}^{2}<1 \%$ ), the risk difference was $8 \%$ in favour of voice notifications $(95 \%$ CI $-16 \%$ to $\left.0.1 \% ; \mathrm{N}=3, \mathrm{p}=0.05, \mathrm{I}^{2}=6 \%\right)$. Pooled 'no show' rates were $15 \%$ for electronic text notifications ( $\mathrm{N}=4$, CI $11 \%$ to $20 \%)$ and $13 \%$ for voice notifications $(\mathrm{N}=4$, CI $7 \%$ to $18 \%$ ). The risk ratio was 1.12 (CI 0.90 to $1.38 ; \mathrm{N}=4$, $\left.\mathrm{p}=0.32, \mathrm{I}^{2}<73 \%\right)$, the risk difference was $1 \%(95 \% \mathrm{CI}$ $-2 \%$ to $4 \% ; \mathrm{N}=4, \mathrm{p}=0.35, \mathrm{I}^{2}=70 \%$ ).

\section{DISCUSSION}

This review and meta-analysis demonstrates that electronic text notifications improve appointment attendance and reduce 'no shows'. Notifications improve attendance and reduce 'no shows'. These findings replicate earlier ones, ${ }^{14}$ but we can have more confidence in the results because they were stable even after removing the influence of studies which were at risk of bias. A novel finding is that two or more notifications increased attendance by as much as $19 \%$ over and above sending one notification, and voice notifications may offer slight improvements over text notifications for increasing attendance.

Taking the UK Secretary of State's estimates literally, a $5 \%$ reduction in 'no shows' across the National Health Service (NHS) GPs and hospitals would save the NHS > $£ 45$ million. There may be additional savings gained by sending multiple (as opposed to single) notifications. Almost all NHS services have an electronic text notification system already; these could be adapted to provide an extra notification at little extra cost to accommodate this change.

Some areas, such as mental health, have historically reported high rates of missed appointments, ${ }^{5}$ where people with severe mental illness may miss up to $45 \%$ of scheduled appointments in primary care. ${ }^{46}$ The studies reviewed here suggested that attendance rates for mental health settings were not dissimilar to those in other settings. These studies do not, therefore, reflect the 'normal' clinic attendance known to be lower and therefore suggests that more studies reflecting usual practice are needed. For those clinical areas with poor attendance, text messages may not act in the same way and may need to be adapted. But currently, we do not have any evidence to draw any conclusion. 

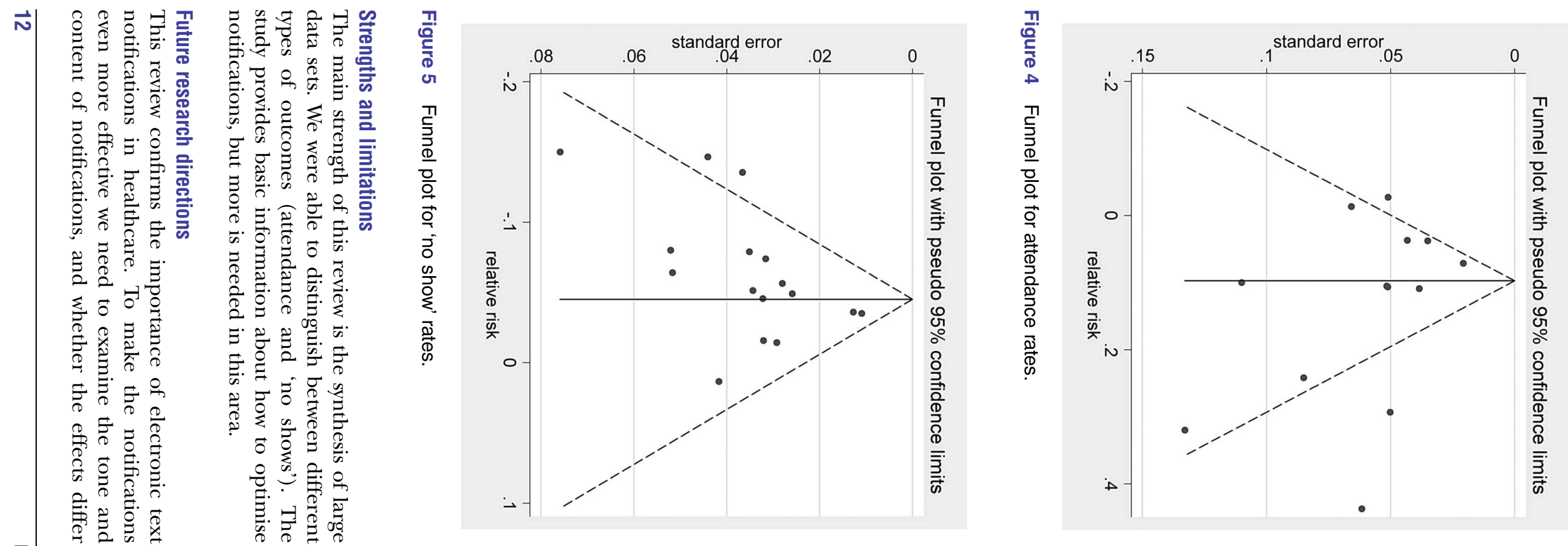

\begin{tabular}{|c|c|c|c|c|c|c|c|c|}
\hline & Intervention (\%) & Control (\%) & Risk ratio & $\begin{array}{l}\text { Risk difference } \\
(\%)\end{array}$ & Coeff* $\left.^{*} \%\right)$ & $\begin{array}{l}\text { p value } \\
\text { (risk } \\
\text { difference) }\end{array}$ & $\begin{array}{l}I^{2} \text { (Risk } \\
\text { difference) (\%) }\end{array}$ & Obs \\
\hline \multicolumn{9}{|l|}{ Attendance } \\
\hline More than one notification & $78(\mathrm{~N}=5, \mathrm{Cl} 68$ to 88$)$ & $52(\mathrm{~N}=5, \mathrm{Cl} 31$ to 78$)$ & $1.49(\mathrm{Cl} 1.17$ to 1.88$)$ & 25 (Cl 11 to 39$)$ & 19 & 0.01 & 66 & 13 \\
\hline $\begin{array}{l}\text { One notification } \\
\text { 'No show' rate }\end{array}$ & $62(\mathrm{~N}=8, \mathrm{Cl}=40$ to 83$)$ & $55(\mathrm{~N}=8, \mathrm{Cl}=31$ to 78$)$ & $1.09(\mathrm{Cl}=1.00$ to 1.18$)$ & $6(\mathrm{Cl}=2$ to 10$)$ & & & & \\
\hline More than one notification & $16(\mathrm{~N}=3, \mathrm{Cl}-3$ to 3$)$ & $24(\mathrm{~N}=3, \mathrm{Cl} 2$ to 46$)$ & 0.75 (Cl 0.57 to 0.99$)$ & $-5(\mathrm{Cl}=-8$ to -1$)$ & 0.3 & 0.91 & 35 & 15 \\
\hline One notification & $15(\mathrm{~N}=12, \mathrm{Cl} 10$ to 20$)$ & $21(\mathrm{~N}=12, \mathrm{Cl} 15$ to 26$)$ & 0.75 (Cl 0.68 to 0.82$)$ & $-5(\mathrm{Cl}-8$ to -3$)$ & & & & \\
\hline
\end{tabular}


between client groups, and what preferences patients have for receiving notifications.

\section{CONCLUSIONS}

Electronic text notifications increase attendance and reduce 'no shows'. Multiple notifications add significantly to the effectiveness. The large number of "no shows' in health services means any successful intervention to reduce them will have cost implications.

Contributors DR, JR and TW designed the study and formulated the clinical question. JR and SS performed the literature search and, with DR, reviewed the search results for study inclusion. DR, JR and SS designed the data extraction form, extracted the data and assessed risk of bias. DS developed the analysis strategy, DR and DS performed the statistical analyses. DR led the writing of the final manuscript with support from SS and JR. All authors critically revised drafts and the final manuscript. All authors approved the final manuscript for submission.

Funding The authors would like to acknowledge the support the National Institute for Health Research (NIHR) Mental Health Biomedical Research Centre at South London and Maudsley National Health Service (NHS) Foundation Trust. The views expressed are those of the authors and not necessarily those of the NHS, the NIHR or the Department of Health.

Competing interests All authors have completed the International Committee of Medical Journal Editors (ICMJE) uniform disclosure form at www.icmje.org/ coi_disclosure.pdf and declare: no support from any organisation for the submitted work; no financial relationships with any organisations that might have an interest in the submitted work in the previous 3 years; no other relationships or activities that could appear to have influenced the submitted work.

Provenance and peer review Not commissioned; externally peer reviewed.

Data sharing statement No additional data are available.

Transparency declaration The lead author affirms that this manuscript is an honest, accurate and transparent account of the review being reported, that no important aspects of the study have been omitted and that any discrepancies from the study as planned (and, if relevant, registered) have been explained.

Open Access This is an Open Access article distributed in accordance with the terms of the Creative Commons Attribution (CC BY 4.0) license, which permits others to distribute, remix, adapt and build upon this work, for commercial use, provided the original work is properly cited. See: http:// creativecommons.org/licenses/by/4.0/

\section{REFERENCES}

1. Torjesen I. Patients will be told cost of missed appointments and May be charged. BMJ 2015;351:h3663.

2. Zailinawati $\mathrm{A}, \mathrm{Ng} \mathrm{C}$, Nik-Sherina $\mathrm{H}$. Why do patients with chronic illnesses fail to keep their appointments? A telephone interview. Asia Pac J Public Health 2005;18:10-15.

3. Martin SJ, Bassi S, Dunbar-Rees R. Commitments, norms and custard creams - a social influence approach to reducing did not attends (Dnas). J R Soc Med 2012;105:101-4.

4. Killaspy $\mathrm{H}$, Banerjee $\mathrm{S}$, King $\mathrm{M}$, et al. Prospective controlled study of psychiatric out-patient non-attendance-characteristics and outcome. Br J Psychiatry 2000;176:160-5.

5. van Dieren Q, Rijckmans MJN, Mathijssen JJP, et al. Reducing no-show behavior at a community mental health center. J Community Psychol 2013;41:844-50.

6. Ericsson. Ericsson Mobility Report: on the pulse of the networked society. Stockholm, Sweden: Ericsson, 2015.

7. Ofcom Communications Market Report [database on the Internet]. 2012. http://consumers.ofcom.org.uk/news/ the-text-message-turns-20-today/

8. Atun RA, Sittampalam SR. A review of the characteristics and benefits of SMS in delivering healthcare. The Role of Mobile Phones in Increasing Accessibility and Efficiency in Healthcare. Vodafone Policy Paper Series Number. 2006;4:18-28.
9. Sims $H$, Sanghara $H$, Hayes $D$, et al. Text message reminders of appointments: a pilot intervention at four community mental health clinics in London. Psychiatr Serv 2012;63:161-8.

10. Car J, Ng C, Atun R, et al. SMS text message healthcare appointment reminders in England. $J$ Ambul Care Manag 2008;31:216-19.

11. Stubbs ND, Geraci SA, Stephenson PL, et al. Methods to reduce outpatient non-attendance. Am J Med Sci 2012;344:211-19.

12. Kannisto KA, Koivunen MH, Valimaki MA. Use of mobile phone text message reminders in health care services: a narrative literature review. J Med Internet Res 2014;16:55-68.

13. Gurol-Urganci I, de Jongh T, Vodopivec-Jamsek V, et al. Mobile phone messaging reminders for attendance at healthcare appointments. Cochrane Database Syst Rev 2013;(12):CD007458.

14. Guy $\mathrm{R}$, Hocking $\mathrm{J}$, Wand $\mathrm{H}$, et al. How effective are short message service reminders at increasing clinic attendance? A meta-analysis and systematic review. Health Serv Res 2012;47:614-32.

15. Smith A, McGeeney K, Duggan M, et al. Mobile access 2010: Pew Internet Reports. Washington DC: Pew Research Centre, 2010.

16. Higgins JP, Green S. Cochrane handbook for systematic reviews of interventions. Wiley Online Library, 2008.

17. Youssef A. Use of short message service reminders to improve attendance at an internal medicine outpatient clinic in Saudi Arabia: a randomized controlled trial. E Mediterr Health $J$ 2014;20:317-23.

18. Everitt B. Modern medical statistics: a practical guide. Arnold, 2003.

19. Tobias A. Assessing the influence of a single study in the meta-anyalysis estimate. Stata Tech Bull 1999;47:15-17.

20. Duval S, Tweedie R. Trim and fill: a simple funnel-plot-based method of testing and adjusting for publication bias in meta-analysis. Biometrics 2000;56:455-63.

21. Moher D, Liberati A, Tetzlaff J, et al. Preferred reporting items for systematic reviews and meta-analyses: the PRISMA statement. Int J Surg 2010;8:336-41.

22. Arora $\mathrm{S}$, Burner $\mathrm{E}$, Terp $\mathrm{S}$, et al. Improving attendance at post-emergency department follow-up via automated text message appointment reminders: a randomized controlled trial. Acad Emerg Med. 2015;22:31-7.

23. Bigna JJ, Noubiap JJ, Kouanfack C, et al. Effect of mobile phone reminders on follow-up medical care of children exposed to or infected with HIV in Cameroon (MORE CARE): a multicentre, single-blind, factorial, randomised controlled trial. Lancet Infect Dis 2014;14:600-8.

24. Bos A, Hoogstraten J, Prahl-Andersen B. Failed appointments in an orthodontic clinic. Am J Orthod Dentofacial Orthop 2005;127:355-7.

25. Chen ZW, Fang LZ, Chen LY, et al. Comparison of an SMS text messaging and phone reminder to improve attendance at a health promotion center: a randomized controlled trial. J Zhejiang Univ Sci B 2008;9:34-8.

26. Cho SJ, Kim YS, Shin $\mathrm{HC}$, et al. A randomized controlled trial of SMS text messaging versus postal reminder to improve attendance after lipid lowering therapy in primary care. Korean $\mathrm{J}$ Fam Med 2010;31:284-93.

27. Clough BA, Casey LM. Using SMS reminders in psychology clinics: a cautionary tale. Behav Cogn Psychoth 2014;42: 257-68.

28. Costa J, Lima M, Sousa D, et al. Impact of appointment reminders via short message service in a district hospital. Actas De La lii Conferencia Iberica De Sistemas Y Tecnologias De La Informacion. 2008;1:557-64.

29. Fairhurst K, Sheikh A. Texting appointment reminders to repeated non-attenders in primary care: randomised controlled study. Qual Saf Health Care 2008;17:373-6.

30. Koury E, Faris C. Mobile phones and clinic appointments: the start of a beautiful new friendship?. Br J Healthcare Comput Info Manag 2005;22:18-20.

31. Leong KC, Chen WS, Leong $\mathrm{KW}$, et al. The use of text messaging to improve attendance in primary care: a randomized controlled trial. Fam Pract 2006;23:699-705.

32. Liew SM, Tong SF, Lee VK, et al. Text messaging reminders to reduce non-attendance in chronic disease follow-up: a clinical trial. Br J Gen Pract 2009;59:916-20.

33. Lin HT, Chen WR, Luo LX, et al. Effectiveness of a short message reminder in increasing compliance with pediatric cataract treatment a randomized trial. Ophthalmology 2012;119:2463-70.

34. Narring F, Perron NJ, Dao MD, et al. Text-messaging to reduce missed appointment in a youth clinic: a randomised controlled trial. $J$ Epidemiol Commun H 2013;67:888-91.

35. Odeny TA, Bailey RC, Bukusi EA, et al. Text messaging to improve attendance at post-operative clinic visits after adult male circumcision for HIV prevention: a randomized controlled trial. PLOS ONE 2012;7:e43832. 
36. Perron NJ, Dao MD, Kossovsky MP, et al. Reduction of missed appointments at an urban primary care clinic: a randomised controlled study. BMC Fam Pract 2010; $11: 79$

37. Prasad S, Anand R. Use of mobile telephone short message service as a reminder: the effect on patient attendance. Int Dent $J$ 2012;62:21-6.

38. Rutland $\mathrm{E}$, Roe H, Weaver A. Health promotional messages in short message service (SMS) follow-up of Gu medicine clinic defaulters; a tool to improve subsequent attendance rates? Sex Transm Infect 2012;88:A4-5.

39. Taylor NF, Bottrell J, Lawler K, et al. Mobile telephone short message service reminders can reduce nonattendance in physical therapy outpatient clinics: a randomized controlled trial. Arch Phys Med Rehabil 2012;93:21-6.

40. Wang KJ, Wang CS, Xi L, et al. A randomized controlled trial to assess adherence to allergic rhinitis treatment following a daily short message service (SMS) via the mobile phone. Int Arch Allergy Imm 2014;163:51-8
41. Fung MK, Briggs B, Frascoia A, et al. The effects of text message reminder on blood donor show rate. Transfusion 2009;49:65A.

42. Nelson TM, Berg JH, Bell JF, et al. Assessing the effectiveness of text messages as appointment reminders in a pediatric dental setting. J Am Dent Assoc 2011;142:397-405.

43. Norton BL, Person AK, Castillo C, et al. Barriers to using text message appointment reminders in an HIV clinic. Telemed E Health 2014;20:86-9.

44. Percac-Lima S, Grant RW, Green AR, et al. A culturally tailored navigator program for colorectal cancer screening in a community health center: a randomized, controlled trial. J Gen Intern Med 2009;24:211-17.

45. Perron NJ, Dao MD, Righini NC, et al. Text-messaging versus telephone reminders to reduce missed appointments in an academic primary care clinic: a randomized controlled trial. BMC Health Serv Res 2013;13:125

46. Akerblad AC, Bengtsson F, Ekselius L, et al. Effects of an educational compliance enhancement programme and therapeutic drug monitoring on treatment adherence in depressed patients managed by general practitioners. Int Clin Psychopharm 2003;18:347-54. 